\title{
Le théâtre de Babylone : nouveauté urbaine et néologisme en Mésopotamie ${ }^{1}$
}

\section{Patrick Michel}

\section{(2) OpenEdition \\ 1 Journals}

Édition électronique

URL : http://journals.openedition.org/edl/111

DOI : 10.4000/edl. 111

ISSN : 2296-5084

Éditeur

Université de Lausanne

\section{Édition imprimée}

Date de publication : 15 mai 2011

Pagination : 153-170

ISBN : 978-2-940331-25-3

ISSN : 0014-2026

\section{Référence électronique}

Patrick Michel, "Le théâtre de Babylone : nouveauté urbaine et néologisme en Mésopotamie " "Études de lettres [En ligne], 1-2 | 2011, mis en ligne le 15 mai 2014, consulté le 21 décembre 2020. URL : http:// journals.openedition.org/edl/111; DOI : https://doi.org/10.4000/edl.111 


\section{LE THÉÂTRE DE BABYLONE: NOUVEAUTÉ URBAINE ET NÉOLOGISME EN MÉSOPOTAMIE ${ }^{1}$}

Même au moment de la plus forte intrusion de l'hellénisme dans l'empire séleucide, l'architecture religieuse conserve un puissant parfum local. C'est dans d'autres domaines de l'urbanisme que s'exprimera l'identité des Séleucides; à Babylone, cela se fera avec la construction d'une palestre et d'un théâtre. On a souvent opposé la ville de Babylone à celle d'Uruk, imaginant que la présence d'un théâtre dans la première faisait d'elle une polis, tandis que la seconde était une ville traditionnellement babylonienne. Une étude de la documentation épigraphique et de quelques éléments de l'urbanisme de Babylone nous permettra de mieux cerner l'identité grecque de la ville. L'apparition d'un théâtre introduira également cet élément dans les textes. Un idéogramme, véritable néologisme, sera créé afin de désigner ce nouveau bâtiment, étranger à la tradition mésopotamienne.

Malgré l'annexion de la Mésopotamie à l'empire séleucide, l'implantation grecque y fut moins marquée que dans la partie occidentale de l'empire. L'architecture religieuse reste traditionnelle et l'identité des rois séleucides s'exprime dans d'autres domaines de l'urbanisation; à Babylone, cela se fera avec la construction d'une palestre et d'un théâtre. Après un rapide survol des données archéologiques à notre disposition concernant le théâtre de Babylone, nous nous attarderons sur l'évolution que suivit ce monument au cours de son histoire. Entre sa construction sous Alexandre le Grand, son abandon durant la période parthe (140 av. J.-C. - 224 apr. J.-C.) et sa restauration au II siècle de

I. R. Van der Spek, "The theatre of Babylon in cuneiform». Cette contribution s'appuie sur les recherches de l'auteur afin de donner une image plus précise de l'hellénisation en Mésopotamie. 
notre ère, c'est un résumé de l'histoire de Babylone qui nous est offert. Cette contribution se propose ensuite de présenter un corpus de textes cunéiformes akkadiens qui concernent directement ce théâtre. Ils permirent à Robartus Van der Spek de mettre en évidence la création d'un néologisme dans la documentation d'époque séleucide. A l'introduction d'un monument nouveau dans la ville correspond l'entrée dans les textes d'un nouveau mot, traduit, semble-t-il, littéralement du grec. Lanalyse de ces textes nous laisse ainsi entrevoir certains aspects de l'utilisation de ce monument par la communauté grecque dans la culture sémitique locale et par là même nous informe sur l'organisation socio-politique de la Babylonie séleucide.

\section{Vestiges archéologiques}

Le théâtre est certainement le monument le plus connu de la Babylone hellénistique. Fouillé par Robert Koldewey en 1904, il ne sera décrit par Alfred Mallwitz qu'en 1954. Orienté vers le sud, il est, comme tous les théâtres grecs, composé d'une orchestra, de gradins, d'une proédrie, de parodoi sans porte dans un premier temps et d'un monument de scène ${ }^{2}$.

Le monument est fait de briques crues avec quelques éléments en pierre, respectant ainsi parfaitement les techniques de construction locales. La cavea dépassant le demi-cercle repose sur la pente naturelle. Au moment de la première fouille, on distinguait seulement cinq gradins au bas du monument, divisés en huit secteurs avec une grande estrade centrale. On estime aujourd'hui à trente-cinq le nombre total de gradins, mais le plan d'ensemble demeure hypothétique. Dans un premier état, l'orchestra avait un rayon de près de $10,90 \mathrm{~m}$, pour un mur de scène de $43 \mathrm{~m}$ de long sur $7 \mathrm{~m}$ de large. Au II $\mathrm{I}^{\mathrm{e}}$ siècle de notre ère, l'orchestra est à nouveau surélevée. Le rayon atteint alors $12,40 \mathrm{~m}$ et à ce stade les parodoi semblent ne pas être couvertes. La cavea a un diamètre de $70 \mathrm{~m}$, tandis que le quadriportique de la palestre a une largeur intérieure de $29 \mathrm{~m}$ pour une profondeur intérieure de $28 \mathrm{~m}$.

Pour suivre les modifications architecturales du théâtre, il convient de rappeler certaines étapes de l'histoire de Babylone après l'arrivée d'Alexandre.

2. Le bâtiment de scène était de type à paraskenia. 
Le théâtre fut construit, sous Alexandre, pour la communauté grecque de la ville. A la création de Séleucie du Tigre sous Séleucos entre 311 et 281 av. J.-C., une part non négligeable des habitants grecs semble avoir quitté la ville pour s'installer dans cette nouvelle capitale. On s'étonne alors des dimensions restreintes du théâtre de Séleucie (Tell Umar) ${ }^{3}$. A ce moment, Babylone n'est plus qu'un centre religieux et la ville occupe une place secondaire après avoir perdu son statut de capitale de l'empire. Les auteurs classiques comme Pline l'Ancien présentent d'ailleurs la fondation de Séleucie comme le début du déclin de Babylone ${ }^{4}$. Les documents épigraphiques nuancent cependant quelque peu cette vision, surtout dès le règne d'Antiochos IV (175-163 av. J.-C.). En effet, ce dernier introduit une nouvelle colonie à Babylone qui semble gagner à nouveau en importance. Le théâtre subit quelques modifications parmi lesquelles on doit peut-être compter la construction du proskenion, indiquant déjà une évolution dans les représentations, à savoir que l'action ne se déroule plus dans l'orchestra, qui est amputée d'une portion pour la construction du mur de scène (fig. 1), mais sur un podium élevé devant la scène. Le proskenion était formé de douze piliers à demi-colonnes et décoré de stuc. Lélévation du monument de scène, dont faisaient partie des éléments en stuc, nous est inconnue. Déjà à cette époque donc, la forme du théâtre évolue. La monumentalité du mur de scène fait d'ailleurs penser aux théâtres de l'époque romaine. Le théâtre subira ensuite une reconstruction partielle au II $\mathrm{e}^{\mathrm{e}}$ siècle de notre ère grâce aux efforts d'évergétisme d'un certain Dioscourides qui voulut sans doute restaurer le monument qui s'était dégradé durant la période parthe.

Le complexe architectural comptait également une palestre (fig. 2), selon un aménagement que l'on rencontre à Séleucie. Sur le plan archéologique, on peut identifier la palestre avec le quadriportique au revers du mur de scène du théâtre. Il est difficile de déterminer la date de sa construction, mais une épigraphe de 109 av. notre ère en atteste déjà l'existence.

Revenons rapidement sur le théâtre de Séleucie du Tigre qui n’a cependant fait l'objet d'aucune publication, chose surprenante quand on connaît le statut de cette ville dans l'histoire de la Mésopotamie hellénistique. Découvert entre 1928 et 1929, il est construit en briques crues et

3. Cf. infra.

4. Pline l'Ancien, Histoire naturelle 6.30. 
Fig. 1 - Théâtre de Babylone avec proskenion.

cuites et possède également un quadriportique derrière le mur de scène. Le diamètre de son orchestra n'est que de $6,50 \mathrm{~m}$, ce qui est extrêmement restreint pour le théâtre d'une capitale; il est d'ailleurs plus petit que celui de Babylone. La taille réduite de ce théâtre surprend lorsque nous savons que Séleucie du Tigre fut la capitale de la Mésopotamie séleucide pendant plusieurs années, alors que Babylone était reléguée au second plan.

\section{Datation}

Comme le relevait Robartus Van der Spek 5 , la datation du théâtre de Babylone n'est pas aisée, mais les travaux d'Alfred Mallwitz tendent à

5. R. Van der Spek, "The theatre of Babylon in cuneiform", p. 445 sq. 
Fig. 2 - Plan général du théâtre de Babylone avec la palestre. 
dater la construction du théâtre du début de la période hellénistique, sous le règne d'Alexandre, lorsque la ville était encore capitale. Une reconstruction suivra au $\mathrm{II}^{\mathrm{e}}$ siècle de notre ère, comme en témoigne cette inscription ${ }^{6}$ sur plaque d'albâtre (fig. 3):

$\Delta \mathrm{IO}$ KKOYPI $[\delta \eta \ldots$

O ФI $\Lambda \mathrm{O} \Delta \mathrm{O} \Xi[\dot{\omega} \tau \alpha \tau \mathrm{\tau}$ ? ...

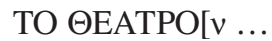

KAI $\Sigma$ KHN[ńv ...

Dioscouri[des ...], montrant du zèle pour la gloire [dans ses dispositions envers la ville/le peuple $[\mathrm{XXX}]$ a réparé ?], le théâtre [...] et le bâtiment de scène $[\ldots]$

La graphie permet de dater l'inscription du $\mathrm{II}^{\mathrm{e}}$ siècle de notre ère, soit de la fin de la période parthe ${ }^{7}$. Les Parthes s'étaient en effet emparés de la ville trois ans après la fin du règne d'Antiochos IV. Sous leur domination, une partie des habitants fut déportée. Quant à la ville, elle aurait été réduite en cendres vers 127 av. J.-C. par le satrape Himeros, qui aurait incendié l'agora et certains temples de Babylone, détruisant une partie de la ville, dont le théâtre.

Malgré ces événements, les chroniques nous indiquent que les activités politiques continuaient à se dérouler à Babylone durant cette période, comme l'atteste le texte présenté plus loin et daté entre le 6 janvier et le 4 février 124 av. J.-C.

On distingue donc principalement trois phases dans l'histoire de ce monument: sa construction sous Alexandre, une première rénovation sous Antiochos IV avec l'introduction probable du proskenion et enfin une reconstruction importante au $\mathrm{II}^{\mathrm{e}}$ siècle de notre ère par Dioscourides. Quant à la palestre, nous savons seulement qu'elle existait en 109 av. J.-C. comme l'atteste l'inscription déjà mentionnée, sans qu'il nous soit possible de savoir à quand remonte exactement sa construction.

6. Ibid., p. 446 et voir aussi F. Wetzel, E. Schmidt, A. Mallwitz, Das Babylon der Spätzeit, p. 21.

7. E. Schmidt, "Die Griechen in Babylon und das Weiterleben ihrer Kultur", p. 843 . 


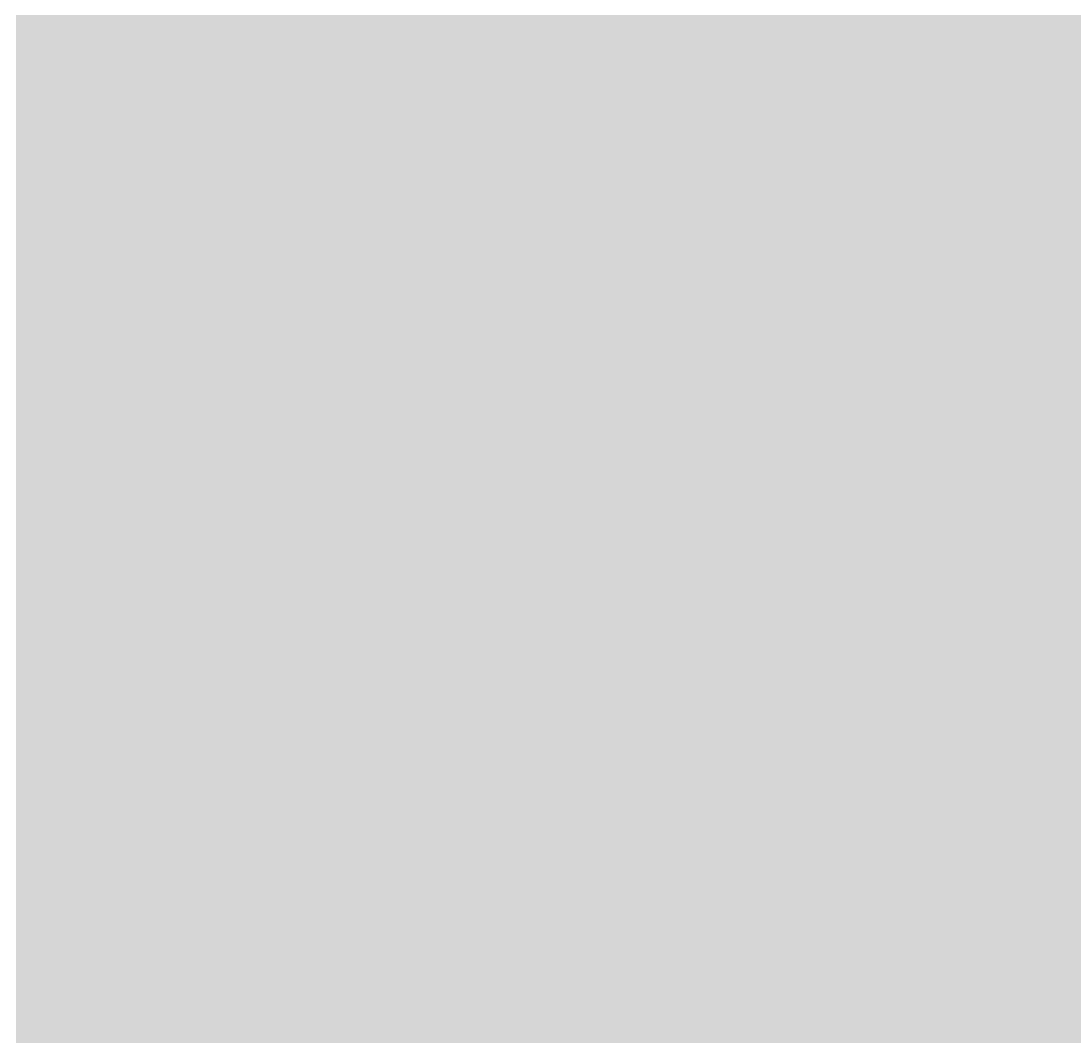

Fig. 3 - Inscription de Dioscourides. Plaque d'albâtre. IIe siècle apr. J.-C.

$(21 \mathrm{~cm} \times 17 \mathrm{~cm})$.

\section{Analyse des sources épigraphiques}

En plus des deux inscriptions grecques déjà mentionnées ci-dessus, qui nous renseignent principalement sur l'histoire du monument, il existe un corpus de tablettes cunéiformes, identifiées sous l'appellation Chroniques astronomiques qui nous éclairent sur l'utilisation du monument. Au maintien de la tradition cultuelle, les temples de Babylone associent en effet, sous les Séleucides et les Parthes, une science astronomique et astrologique qui atteint son apogée à cette époque. Ces textes sont donc d'une grande importance puisqu'ils nous aident à mieux connaître la situation politique et sociale de la ville et de sa région durant la période gréco-parthe, nuançant quelque peu notre perception de l'impact des exactions du tyran Himeros. Il s'agit par ailleurs des seules sources nous 
permettant de juger réellement de l'effet de la présence grecque sur le quotidien des Babyloniens.

Ces textes font régulièrement référence aux membres d'une communauté grecque de Babylone dénommée en akkadien sous la forme (donnée ici syllabiquement) $p u-l i-t e-e$, mot qui n'est que la transcription en cunéiforme akkadien du grec politai.

5 janvier - 2 février 132 av. J.-C.

Ce mois, un messager du roi est entré à Babylone. Le šatammu de l'Esagila et les Babyloniens de la kiništu de Babylone ont fourni un bœuf et cinq moutons pour ce messager du roi. A Bel, Beltia, et les grands dieux et pour la vie du $\mathrm{roi}^{8}$ et sa propre vie, il les sacrifia. Ce mois, une lettre parchemin, écrite à l'epistates et aux politai, qui sont à Babylone, a été lue dans la maison de l'observation ${ }^{9}$ comme suit... ${ }^{10}$

Ce texte confirme la coexistence de deux communautés dans la ville de Babylone: les Babyloniens et les Grecs. Nous apprenons que le messager arrivant à Babylone est en premier lieu allé dans la communauté babylonienne où il a fait des offrandes, puis il s'est rendu dans la communauté grecque où la lettre a été lue dans le théâtre.

Ce corpus de textes nous renseigne surtout sur l'organisation politique de la communauté grecque qui n'était pas dirigée par la même autorité que la population babylonienne et qui formait une communauté à part dans la ville. La communauté des Babyloniens, qui se nommait «fils de Babylone ", transcrit en akkadien mār Bäbili, ou en employant la notation sumérienne logographique DUMU.MEŠ É.KI, était dirigée par le šatammu, président de l'assemblée, kiništu. L'assemblée et son président, issus des temples, se réunissaient dans le bit milki « la maison de la délibération ». De son côté, la communauté grecque était placée sous l'autorité d'un pahatu (traduction du grec epistates ${ }^{11}$ ). Il arrivait que ce groupe se réunît afin d'écouter le message, sous forme de lettre, délivré par le roi

8. La question d'un culte royal séleucide en Mésopotamie est encore sujette à discussion, cf. P. Michel, M. Widmer, «Entre l'Asie Mineure et la Babylonie: Regards croisés sur le culte royal séleucide».

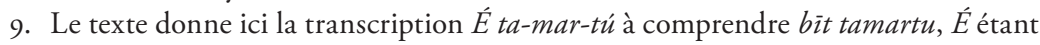
le logogramme sumérien pour «maison».

IO. A. J. Sachs, H. Hunger, Astronomical diaries and related texts from Babylonia, III, p. 230.

II. G. F. Del Monte, Testi della Babilonia ellenistica, I, p. 96 sq. 
et les gouverneurs pour le pahatu et les politai. Le lieu de réunion de ces Grecs était la bìt tāmartu transcrit logographiquement É.IGI.DU .MEŠ, $^{-}$ terme qu'on retrouve plusieurs fois dans les Chroniques astronomiques. Littéralement, on pourrait traduire l'expression par «maison de l'observation». Pour Robartus Van der Spek que nous suivons, cette «maison de l'observation" est en fait le théâtre de Babylone. C'est ce que nous indique encore une chronique datée du 16 février au 17 mars 87 av. J.-C.:

Ce mois [XXX], le $3^{\mathrm{e}}$ jour, leurs lettres ont été lues dans le théâtre ${ }^{12}$, comme suit: Le roi Arsaces, dont le nom est Gotarzes, les a battus $[\mathrm{XXX}]$ il a pris possession.

A Babylone, le théâtre était donc le lieu de réunion des politai lorsque le roi ou les gouverneurs s'adressaient à eux. Les mêmes lettres du roi étaient lues séparément, en des lieux différents comme on l'a vu, à la communauté babylonienne et à la communauté grecque ${ }^{13}$.

Le nom du bâtiment, qui apparaît soit sous sa forme phonétique akkadienne soit sous la forme idéographique, est construit à partir du mot bit «maison» et tāmartu, du verbe amāru "voir», «regarder». Le même phénomène se vérifie en grec. On sait en effet que le terme $\theta \dot{\varepsilon} \alpha \tau$ cov «théâtre»

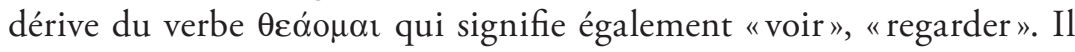
semble ici que le terme akkadien soit le fruit d'une traduction littérale du mot grec. Ce phénomène nous renseigne donc sur la présence à Babylone de scribes maîtrisant parfaitement l'emploi du grec et de l'akkadien. Ce fait est confirmé d'ailleurs depuis la découverte des Graeco-babyloniaca, un corpus de textes où l'akkadien est transcrit phonétiquement en caractères grecs ${ }^{14}$. L'introduction d'un monument nouveau et de surcroît étranger à la culture locale dans la ville de Babylone donna ainsi naissance à un néologisme dans la documentation épigraphique akkadienne.

Le théâtre était un lieu de réunion idéal, pouvant accueillir de nombreuses personnes qui, une fois rassemblées, pouvaient écouter les

I2. Le texte nous donne ici la transcription logographique sumérienne É IGI.DU I $^{\text {A }}$ qu'on traduit désormais par théâtre.

13. A. J. Sachs, H. Hunger, Astronomical diaries and related texts from Babylonia, III, p. 454.

I4. Précisons que ces textes ont d'ailleurs permis de s'assurer de certaines lectures et prononciations phonétiques akkadiennes. Une présentation de ce corpus est consultable en ligne à l'adresse suivante: http://www.unige.ch/lettres/antic/mesopotamie/ graecobabyloniaca.html (dernière consultation novembre 2010). 
discours sans grande difficulté grâce à une bonne acoustique. Il semble évident qu'il s'agissait du meilleur monument pour s'adresser à une foule. Le phénomène est connu également à Athènes durant la période hellénistique. Le discours que prononça le philosophe Athénion, cité par Athénée, le prouve. Ayant pour but de rallier les Athéniens du côté de Mithridate lors de la lutte contre les Romains en 87-86 av. J.-C., ce discours se présente comme suit:

Ne tolérez plus l'état anarchique de la situation, que le Sénat romain a étendu jusqu'à maintenant comme s'il devait nous indiquer le type de gouvernement à adopter. Ne permettez pas que nos lieux sacrés nous soient fermés [...] et nos théâtres désertés par l'assemblée... ${ }^{15}$

Le théâtre d'Athènes était donc devenu le lieu de réunion tandis que le Pnyx s'était doté d'une importance religieuse. Un certain nombre d'inscriptions honorifiques précisent de plus qu'un décret a été fait durant une assemblée dans le théâtre ${ }^{16}$. Cet autre document, bien que lacunaire, nous donne également des informations intéressantes pour notre propos:

5 avril - 4 mai 162 av. J.-C.

L'epistates de Babylone et les politai qui sont à Babylone, avec Nabumušetiq-uddi, fils de [XXX], le šatammu de l'Esagila et ses frères donna [aux?] politai. Devant les généraux et les juges [XXX] et deux [XXX], qui dans le passé [XXX] Esagila et le palais du roi sont retournés et se sont présentés à la cour devant l'epistates $[\mathrm{XXX}]$ et ses deux frères, dont l'un a été tué et ses biens confisqués au profit du trésor royal $[\mathrm{XXX}]$. Les gens du pays ont été interrogés sous la menace (?) sur le petit côté (?) et la maison de l'observation (le théâtre) qui se trouve dans le district $[\mathrm{XXX}]^{17}$.

Soulignons ici le fait que l'epistates (pahatu) est nommé en lien avec les politai et qu'il représente le pouvoir en place puisqu'il dirige la cour avec les juges. Enfin, un lieu proche ou directement contre le théâtre est désigné comme lieu de réunion pour le tribunal. Le conseil des Anciens dans

15. Athénée de Naucratis, fragment 87 édité par F. Jacoby, Die Fragmente der griechischen Historiker.

I6. SEG 38, $1988 \mathrm{n}^{\text {os }} 108$ et 115 .

I7. A. J. Sachs, H. Hunger, Astronomical diaries and related texts from Babylonia, III, p. 30. 
le théâtre se nomme peliganes. Il s'agit d'un nom macédonien transcrit en akkadien avec la marque du pluriel: pe-li-ga-na-nu. Ce conseil était supervisé par un gouverneur qui portait le nom de pahatu.

Nous citons enfin un extrait important datant de la période parthe:

6 janvier - 4 février 124 av. J.-C.

Ce mois, une lettre parchemin de Hyspaosines, roi de Mésène, qui a écrit au général de Babylone, a été amenée ici et lue aux politai qui sont à Babylone, comme suit:

«En ce mois, le $15^{\mathrm{e}}$ jour, le roi Arsaces et Pittit, l'ennemi Elamite, se sont battus. Le roi a défait les troupes des Elamites en bataille. Il a pris Pittit.»

Ce mois, le $7^{e}$ jour, une $[\mathrm{XXX}]$ a donné naissance et le nouveau-né était comme $[\mathrm{XXX}]$ il avait le $[\mathrm{XXX}]$ d'un chien. Ce mois, le $15^{\mathrm{e}}$ jour, le trône royal, qui suivant les plans d'un nouveau modèle en bois et en argent $[\mathrm{XXX}]$ a été fait, dont le nom chez les Grecs est thronos, que le roi Hyspaosines, dans le passé, avait pris dans le Palais royal à Babylone et l'avait offert en cadeau à Bel, l'epistates de Babylone et les Grecs de la ville [verbe], et ils ont ouvert les portes du temple du Nouvel-An, ils ont fait [XXX], mais ils ne sont pas entrés. Le trône royal en question, le cadeau fait à Bel, ils l'ont déplacé et pris pour eux. Ce mois, un messager du roi portant un parchemin est entré à Babylone. Ce jour, la lettre du roi, écrite à l'attention de l'epistates de Babylone et pour les Grecs de la ville, a été lue dans le théâtre comme suit: «bataille avec Pittit, l'ennemi de l'Elam, j'ai fait, et j'ai conquis 15000 de ses troupes, dans mes rangs aucune perte. J'ai frappé l'Elam dans sa totalité, Pittit l'ennemi élamite, je l'ai saisi.» Ce jour, le šatammu de l'Esagila et les Babyloniens du kiništu de l'Esagila, ont offert un bœuf et deux moutons à la porte du Fils du Prince de l'Esagila en offrande pour le messager du roi et à Bel et Belita, les grands dieux, ils les ont sacrifiés. Le message du roi a été lu ${ }^{18}$.

Ce texte est particulièrement intéressant. Cette chronique montre la séparation qui existait entre les Grecs et les Babyloniens. C'est avec animosité certainement que l'astronome raconte comment les Grecs ont pris le trône, cité d'ailleurs sous l'appellation grecque, hors du temple, bien que Hyspaosines, lors de son court règne en 127 av. J.-C., l'ait offert à Bel. Il s'agit d'un acte impie et d'un sacrilège commis par les Grecs de

I8. Ibid., p. 278. 
la ville. On ne connait pas les motivations des Grecs, mais on peut penser qu'ils n'appréciaient pas que cet objet, vraisemblablement d'origine grecque comme son nom l'indique, ait été offert au temple pour la population akkadienne alors que sa place initiale était dans le Palais royal. Certains textes témoignent ainsi d'une hostilité certaine entre Grecs et Babyloniens. Cela n'empêche cependant pas les membres des deux communautés de travailler et de commercer ensemble, comme l'attestent de nombreux contrats.

On peut également noter que les Grecs reçoivent un même message de deux rois différents et qui mentionne que le roi Artabane II a vaincu les Elamites. Il s'agit donc bien d'une victoire parthe dont on annonce la nouvelle une fois pour les Grecs dans le théâtre et une fois dans la maison de la délibération pour les Babyloniens. Ainsi la pratique de la lecture séparée des lettres royales est-elle clairement attestée. L'étude de ces textes nous montre également qu'à cette date la ville de Babylone ne semble ni désertée ni incendiée, mais qu'au contraire, toutes les activités quotidiennes y ont encore leur place. Les exactions du tyran Himeros, qui cessa de sévir vers 127 av. J.-C., ne devaient pas avoir été si grandes que les sources nous le laissent croire.

\section{L'assemblée grecque de Babylone et la Boulè}

Ces textes posent la question de l'assimilation de cette assemblée de Grecs à la Boulè. Pour rappel, la Boulè est, dans les cités grecques, le nom du conseil chargé du gouvernement de l'Etat. C'est un corps de citoyens spécialement nommés qui appliquaient les décisions de l'assemblée souveraine et veillaient au fonctionnement quotidien de l'Etat. Dans l'Athènes classique, le conseil comportait cinq cents citoyens masculins de plus de trente ans, tirés au sort chaque année parmi les volontaires après la réussite d'un examen préliminaire. L'origine de la Boulè remonte aux réformes de Clisthène à la fin du $\mathrm{VI}^{\mathrm{e}}$ siècle av. J.-C. Le conseil avait deux fonctions principales, à savoir superviser l'activité des magistrats et assumer le travail administratif, en se réunissant en sessions plénières ou grâce à des comités.

La Boulè préparait les ordres du jour pour les réunions de l'ekklêsia (assemblée). Elle ne dirigeait cependant pas pour autant la politique, même si elle rédigeait sans doute des décrets sur des points qui 
ne prêtaient pas à discussion. Elle siégeait tous les jours, sauf les jours de fête. Pendant les révolutions oligarchiques de 411 à 404 av. J.-C., la Boulè fut remplacée par des conseils oligarchiques, mais elle fut rétablie avec la démocratie et continua à fonctionner tout au long des périodes hellénistique et romaine.

Qu'en est-il de la Boulè en Mésopotamie séleucide? Le cas d'Uruk est très éclairant à ce sujet. Cette ville du sud de la Mésopotamie est une polis grecque où l'assemblée du peuple, la seule et unique que nous connaissions en Mésopotamie, est structurellement proche de la Boulè des cités grecques. A Uruk, les textes attestent une seule assemblée civile pour la population. Les citoyens sont nommés Urukaja, soit "ceux d'Uruk", et l'assemblée qui, avant la période séleucide, n'était qu'une assemblée du temple, est devenue une assemblée civile. Cette situation différente s'explique sans doute par le fait qu'il n'existait pas, près d'Uruk, d'autre centre politique ou culturel ${ }^{19}$, tandis que près de Babylone avait été fondée la nouvelle capitale de Séleucie du Tigre, de sorte que Babylone ne pouvait plus être le centre politique et culturel. Dans ces conditions, la taille restreinte du théâtre de Séleucie pose un certain nombre de questions. Si le centre politique était Séleucie, pourquoi ne pas avoir doté la ville d'un grand théâtre, au moins aussi grand que celui de Babylone? Le cas du théâtre de Babylone est à cet égard tout à fait singulier. Il ne servait de lieu de réunion que pour les Grecs de la ville, cependant sa taille est largement supérieure à celui du centre politique le plus proche. La seule assemblée connue à Babylone est celle du temple de l'Esagila, placée sous une autorité religieuse. Il s'agit donc d'une assemblée religieuse et jamais d'une assemblée civile.

Nous trouvons donc deux cas de figure distincts qui s'expliquent par la présence ou non d'un centre politique grec dans ou près de la ville. On note justement qu'à Uruk l'assemblée est séculaire, elle ne s'occupe que de loin des affaires du temple. A Babylone, quoi qu'il en soit, les institutions des temples étaient purement babyloniennes et nullement le résultat d'influences helléniques. Quant à la communauté grecque de Babylone, il semble en effet que l'organisation était bien celle connue

I9. On a discuté l'existence d'une colonie d'Antiochia près d'Uruk, tout comme celle de Séleucie proche de Babylone, pour expliquer le manque de preuve d'influences helléniques dans l'architecture de la ville d'Uruk. Il est possible qu'Antiochia ne soit autre qu'Uruk même. 
dans les villes grecques. Les Grecs fonctionnaient à Babylone comme dans n'importe quelle cité grecque et la ségrégation dont les textes font écho ne semble que confirmer cet état de fait.

Mais cet aspect particulier de l'utilisation du théâtre de Babylone ne doit pas nous faire oublier sa fonction première, à savoir un lieu des représentations artistiques et théâtrales. Le théâtre de Babylone dut certainement accueillir des concours musicaux et des représentations théâtrales durant la période hellénistique, puis, au $\mathrm{II}^{\mathrm{e}}$ siècle de notre ère, des mimes, des pantomimes et des spectacles de danse. Mais la documentation épigraphique à notre disposition ne nous le confirme malheureusement pas.

\section{Conclusion}

Le théâtre de Babylone est une exception dans le monde syromésopotamien. Dans quel sens? Je préciserai seulement qu'en Syrie, aucun théâtre ne présente, ni dans sa situation, ni dans sa structure, de trace d'un plan ou d'élévation préexistants. On ne relève aucune trace hellénistique précise en Syrie, contrairement aux théâtres de l'Asie Mineure. Il semblerait donc, et Edmond Frézouls ${ }^{20}$ le remarquait déjà, que c'est dans les régions qui ne furent jamais romanisées, mais conquises par Alexandre, qu'on retrouve des théâtres de type grec. Nous ne possédons pas de preuve, me semble-t-il, attestant, en Syrie, l'existence de théâtres datant de la période allant d'Alexandre au début de l'époque romaine. Antioche n'a fourni aucune trace de son théâtre et l'existence présumée de ce dernier repose uniquement sur des hypothèses et conjectures motivées par l'importance de la ville hellénistique. En effet, tous les théâtres connus au Levant sont attribuables au plus tôt à l'époque augustéenne.

Cette rencontre se voulait également l'occasion d'étudier la fonctionnalité d'un monument comme le théâtre. Je pense que notre contribution aura ouvert une porte sur un aspect moins connu de l'utilisation des théâtres, mais qui semble s'être répandu durant la période hellénistique comme nous l'avons vu: le théâtre comme lieu de réunion. La méthode d'investigation suivie a été une démarche d'épigraphiste plus

20. E. Frézouls, «Aspects de l'histoire architecturale du théâtre romain». 
que d'archéologue. Mêlant ainsi l'archéologie à l'étude des sources, nous avons pu donner une image plus complète du rôle du théâtre comme lieu de la vie sociale, mais aussi comme centre de la vie politique d'une communauté grecque dans la capitale d'Alexandre.

Patrick Michel Unité de langues et civilisations de Mésopotamie Université de Genève 


\section{BIBLIOGRAPHIE}

\section{Abréviation}

SEG Supplementum Epigraphicum Graecum, Leiden, 1923-1971, puis Amsterdam, 1979-.

\section{Sources}

Jaсову, Felix, Die Fragmente der griechischen Historiker, Leiden, Brill, 1995.

Pline l'Ancien, Histoire naturelle, livre VI, Paris, Dubochet, Emile Littré (éd.), 1848-1850.

\section{Etudes}

Del Monte, Guiseppe F., Testi della Babilonia ellenistica, vol. 1 : Testi cronografici, Pisa/Roma, Istituti Editoriali e Poligrafici Internazionali, 1997.

FrÉzouls, Edmond, "Aspects de l'histoire architecturale du théâtre romain", Aufstieg und Niedergang der Römischen Welt, 2.12.1 (1982), p. 343-441.

Koldewey, Robert, Das wieder erstehende Babylon, Zürich, Olms, 1981. Michel, Patrick, Widmer, Marie, "Entre l'Asie Mineure et la Babylonie: Regards croisés sur le culte royal séleucide", XVII International Congress of Classical Archaeology: Meetings between cultures in the Ancient Mediterranean, Rome, 22-26 septembre 2008, poster inédit.

Sachs, Abraham Joseph, Hunger, Hermann, Astronomical diaries and related texts from Babylonia, vol. III. Diaries from 164 BC 
to $61 B C$, Wien, Verlag der Österreichischen Akademie der Wissenschaften, 1996.

Schmidt, Erich, "Die Griechen in Babylon und das Weiterleben ihrer Kultur", Archaeologische Anzeiger, 56 (1941), p. 786-844.

VAN Der SpeK, Robartus, "The theatre of Babylon in cuneiform", in Veenhof Anniversary Volume. Studies Presented to Klas Veenhof on the occasion of his sixty-fifth Birthday, ed. Wilfred H. van Soldt, Leiden, Eisenbrauns, 2001, p. 445-456.

Wetzel, Friedrich, Schmidt, Erich, Mallwitz, Alfred, Das Babylon der Spätzeit, Berlin, Mann, 1957.

\section{Crédits iconographiques}

Fig. 1 et 2 :

Wetzel, Friedrich, Schmidt, Erich, Mallwitz, Alfred, Das Babylon der Spätzeit, Berlin, Mann, 1957, pl. 9 et 11a.

Fig. 3:

Koldewey, Robert, Das wieder erstehende Babylon, Zürich, Olms, 1981, p. 293. 
